\title{
Urgent Endovascular Treatment of Iliac Artery Pseudoaneurysm in Patient with Ehlers-Danlos Syndrome: A Case Report
}

\author{
Aleksandar Gjoreski ${ }^{1 *}$, Ivona Jovanoska ${ }^{1}$, Gorgi Dungevski ${ }^{1}$, Nikola Lazovski ${ }^{2}$, Menka Lazareska $^{3}$ \\ ${ }^{1}$ Department of Diagnostic and Interventional Radiology, City General Hospital "8 8 "September", Skopje, Republic of Macedonia; \\ ${ }^{2}$ University Clinic of Vascular Surgery, "St. Cyril and Methodius" University, Skopje, Republic of Macedonia; ${ }^{3}$ University Clinic \\ of Radiology, "St. Cyril and Methodius" University, Skopje, Republic of Macedonia
}

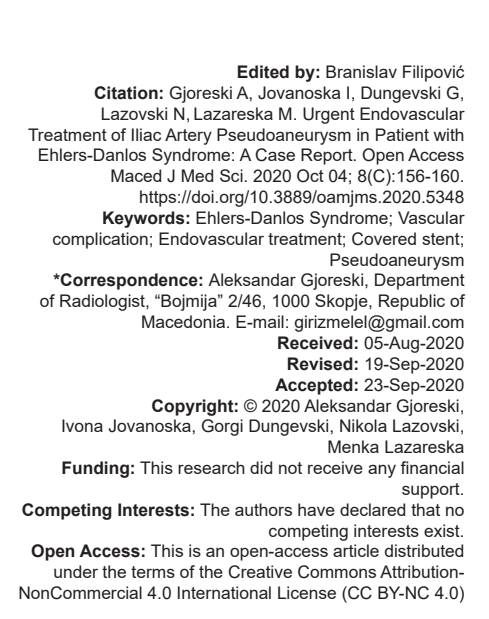

\begin{abstract}
BACKGROUND: Ehlers-Danlos syndrome (EDS) type IV is a heritable disorder of connective tissue that is mainly associated with vascular maladies such as aneurysms, pseudoaneurysms, and dissections with or without spontaneous rupture. Historically, vascular complications in EDS IV have been treated conservatively whenever possible, due to the high morbidity and mortality after vascular interventions, whether open or endovascular. We present a case of a ruptured pseudoaneurysm of the right common iliac artery in a 18-year-old male, who was successfully treated by endovascular approach and later diagnosed with EDS type IV.

CASE PRESENTATION: A 18-year-old male patient was admitted in ER with sharp pain in the right hypogastrium, hypotensive and with reduced blood parameters. Multiphasic modern computed tomography (MDCT) scan of abdomen and pelvis revealed massive ride sided pelvic and retroperitoneal hematoma. The presence of pseudoaneurysms on both common iliac arteries ( $\mathrm{ClA}$ ) was detected, with small ulcer on the right side and a focal dissection on the left side. An urgent endovascular repair of the ruptured pseudoaneurysm on the right CIA with covered stent was performed. Patient's laboratory parameters and clinical status improved significantly within the next few days.

CONCLUSIONS: Vascular repair in EDS-IV patients carries significant risk and should be indicated very carefully. Endovascular treatment for these patients is feasible and should be considered as an alternative to open surgery in some challenging cases as this one.
\end{abstract}

\section{Background}

Ehlers-Danlos syndrome (EDS) is a heterogeneous group of heritable disorders of connective tissue with many subtypes. Type IV subtype results from an autosomal dominant mutation in the COL3A1 gene that causes a defect of type III collagen production with current prevalence of 1 in 50,000-90,000. Extensive bruising, history of vascular maladies (aneurysms, pseudoaneurysms, and dissections with or without spontaneous rupture), spontaneous intestinal perforation, and uterine rupture are ones of the major characteristic clinical findings in patients with EDS-IV [2]. The overall life expectancy of these patients is dramatically shortened with a median life span of 48 years.

A disease-related complication affects $25 \%$ of them by age 20 and $80 \%$ by age 40 [1]. Vascular complications very often present emergently secondary to vascular rupture and without previous diagnosis of the underlying genetic condition.

Historically, vascular disease in EDS-IV has been treated conservatively whenever possible, favoring surgery as an option in life-threatening situations. The reported operative mortality including emergent cases ranges from $7 \%$ to $41 \%$ [3].

We presentacase ofaruptured pseudoaneurysm of the right common iliac artery in a 18-year-old male, who was successfully treated by endovascular approach and later diagnosed with EDS type IV.

To the best of our knowledge, so far one case of elective endovascular treatment for an iliac artery aneurysm in a patient with EDS-IV has been reported in the international literature [4].

\section{Case Presentation}

A 18-year-old male patient was admitted in ER with sharp pain in the right hypogastrium which lasted for almost 2 days and it worsened over time. At time of admission, he was hypotensive and with reduced blood parameters. Physical examinations revealed a normal weight youngster, pale skin, and with no evident 

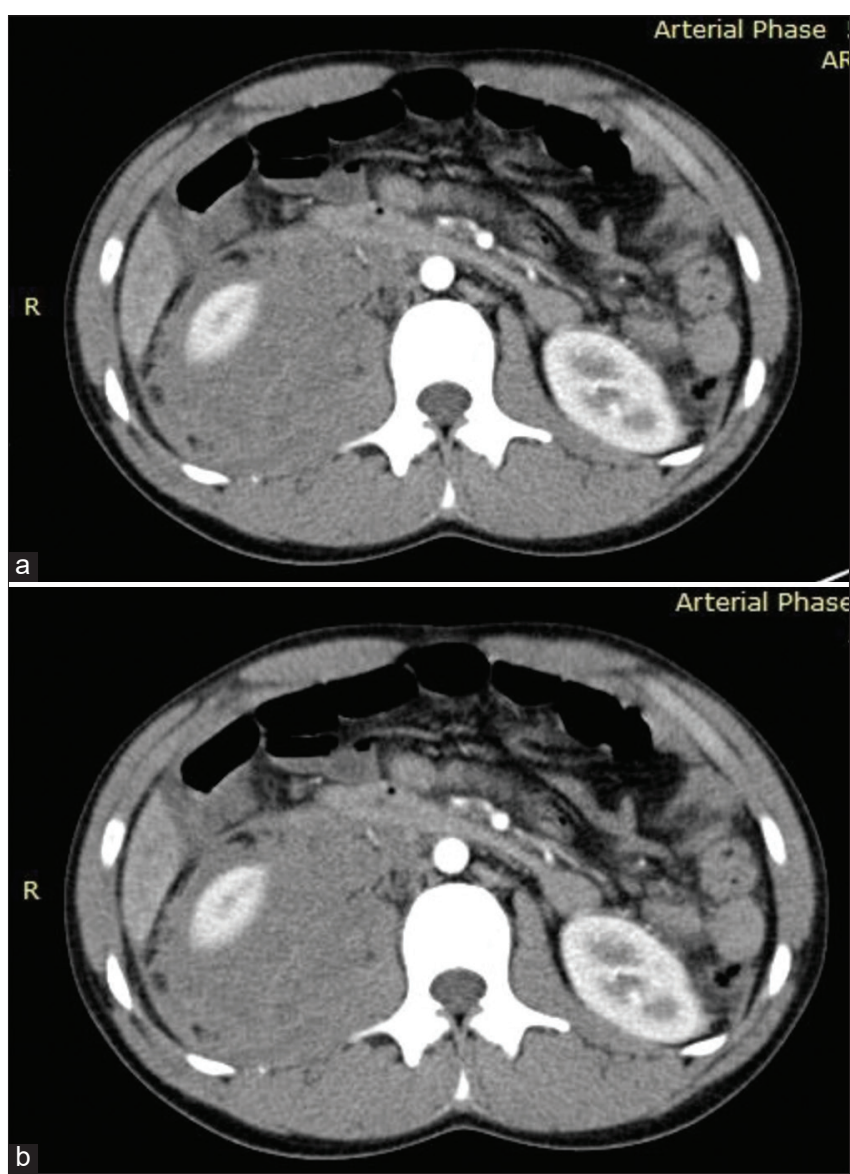

Figure 1: Preprocedural CT scan in arterial phase (a) and (b) showing massive right sided retroperitoneal and pelvic hematoma

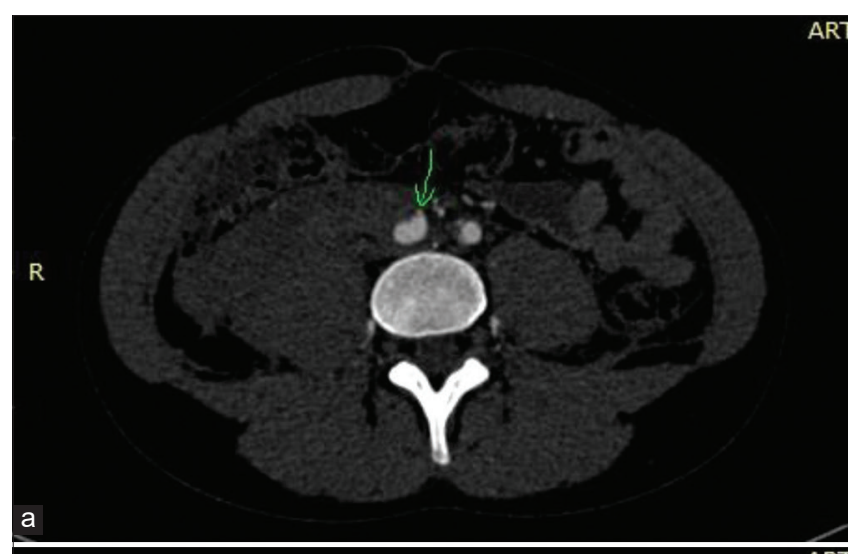

ART

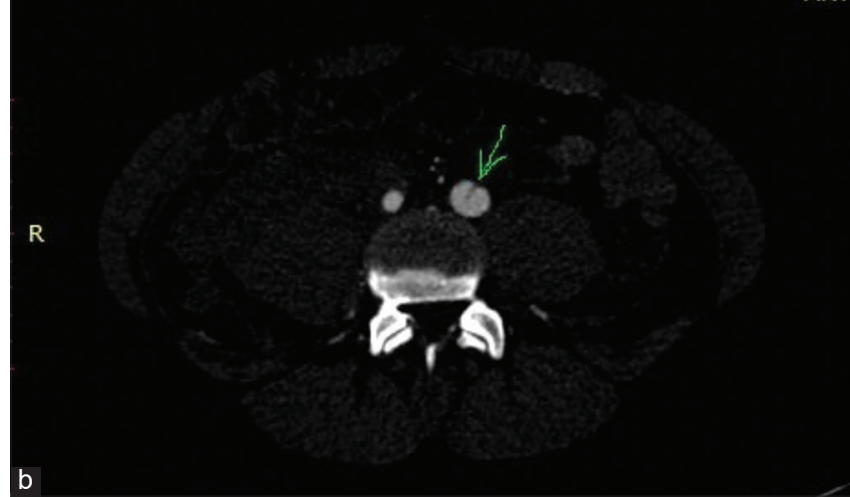

Figure 2: Axial CT scan in arterial phase (a) small pseudoaneurysm of the right CIA with active bleeding and (b) large pseudoaneurysm with local dissection of the left CIA

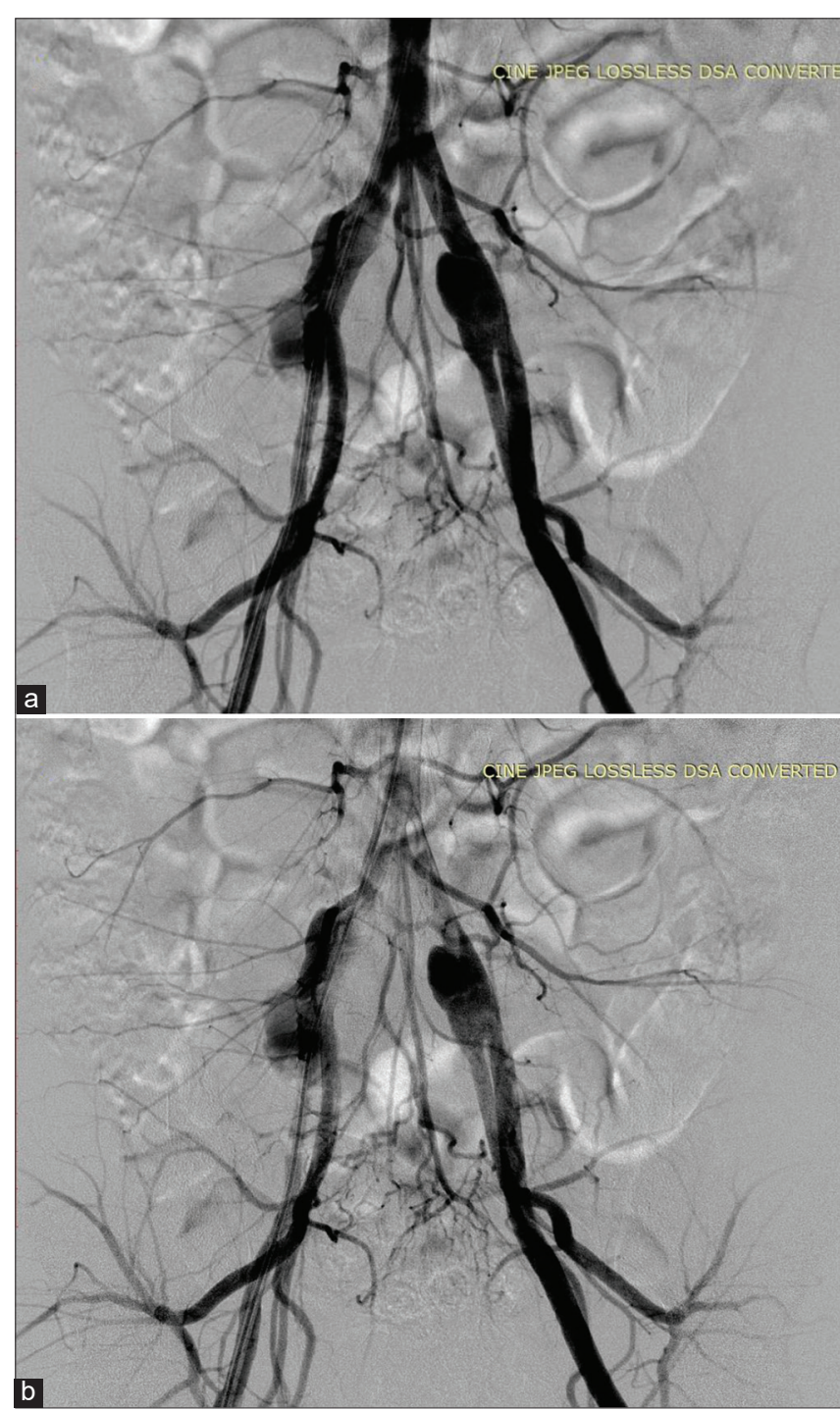

Figure 3: Catheter directed pigtail angiography (a) early arterial and (b) late arterial phases showing active bleeding from right the CIA

dysmorphism. No recent trauma or surgeries were reported. Family history was significant for maternal death after labor due to abdominal hemorrhage.

Multiphasic MDCT scan of abdomen and pelvis was performed, which revealed massive ride sided pelvic and retroperitoneal hematoma (Figure 1).

On the arterial phase presence of pseudoaneurysms on both CIAs was detected, with small ulcer on the right side and a focal dissection on the left side (Figure 2).

The pseudoaneurysm on the right $\mathrm{CIA}$ was suspected as a possible target location for the active bleeding. In light of this active bleeding and continuous drop of RBC, HGB, and HCT as well as hypotension, urgent treatment was indicated. Multidisciplinary vascular team decided that endovascular treatment of the pseudoaneurysm of the right CIA is the least invasive and most appropriate approach. After careful pre-procedural reviewing of the CT images, we decided not to cover the ostium of right internal iliac artery to make the procedure as simple as possible. 


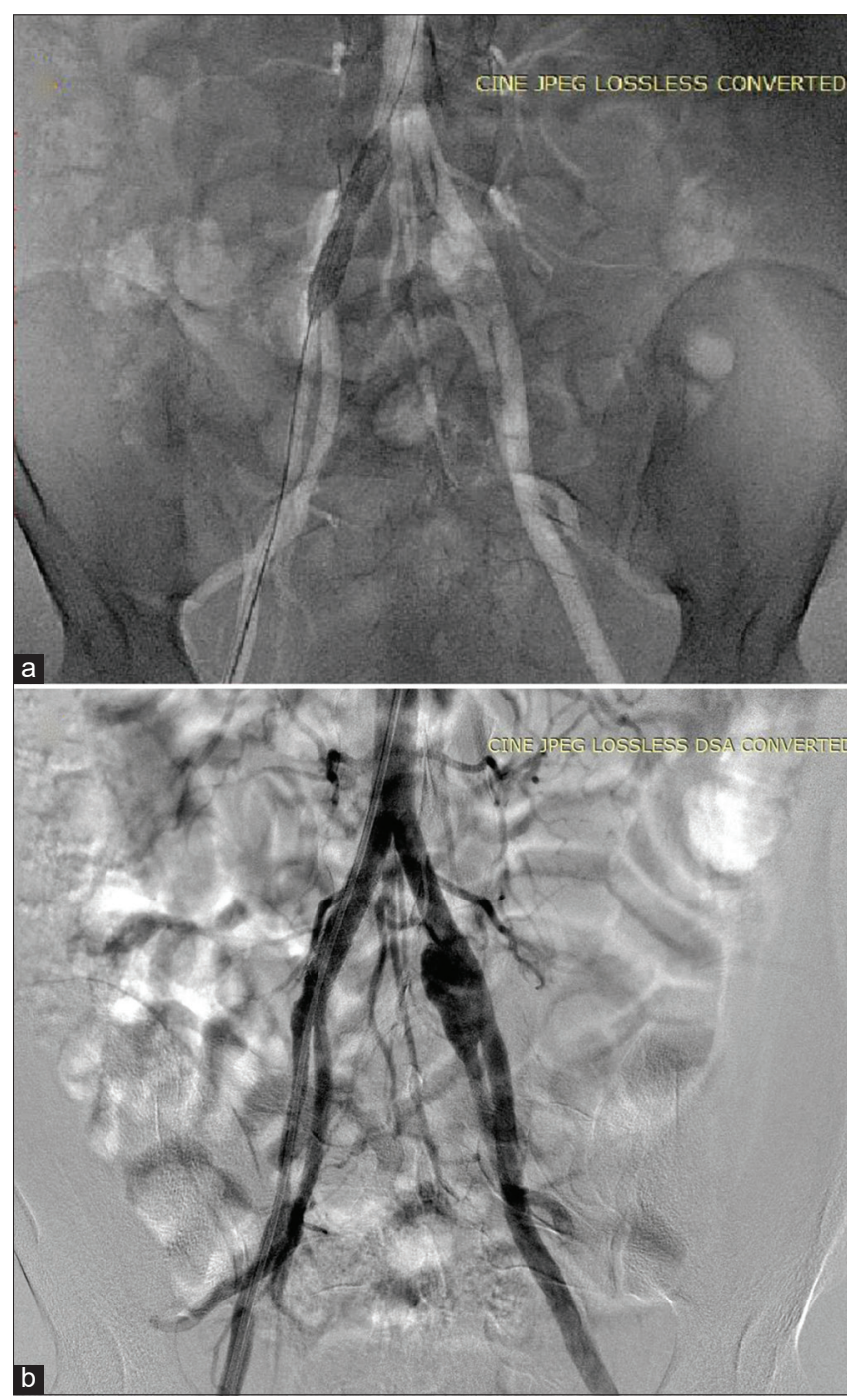

Figure 4: (a) Placement of balloon expandable stent graft $9 \square 38$ mm (Advanta V12, Getinge) under RM fluoroscopy and (b) contrast aortoiliac angiography showing good position of stent graft with complete resolution of bleeding

The intervention was conducted with conscious sedation and local anesthesia. Right common femoral approach was obtained, and a 7F introducer (Radiofocus, Terumo) was placed. Aortoiliac angiography with a $5 \mathrm{~F}$ pigtail catheter placed in the distal abdominal aorta was performed and an massive, active bleeding from right CIA was confirmed (Figure 3).

An ultra-stiff hybrid guidewire (Advantage 0.035," $260 \mathrm{~cm}$, Terumo) was crossed in thoracic aorta. After a predilatation with $7 \times 40 \mathrm{~mm}$, non-compliant balloon catheter (Mustang, Boston Scientific), a $9 \times 38 \mathrm{~mm}$ balloon expandable stent graft was deployed over the pseudoaneurysm (Advanta V12, Getinge group) (Figure 4).

Control angiography showed no residual bleeding with complete patency of CIA and IIA. Puncture site hemostasis was perfumed with manual compression. Patient was discharged from hospital on second post-procedural day.

On first post-intervention day patient's laboratory parameters stabilized and his clinical status improved significantly within the next few days.

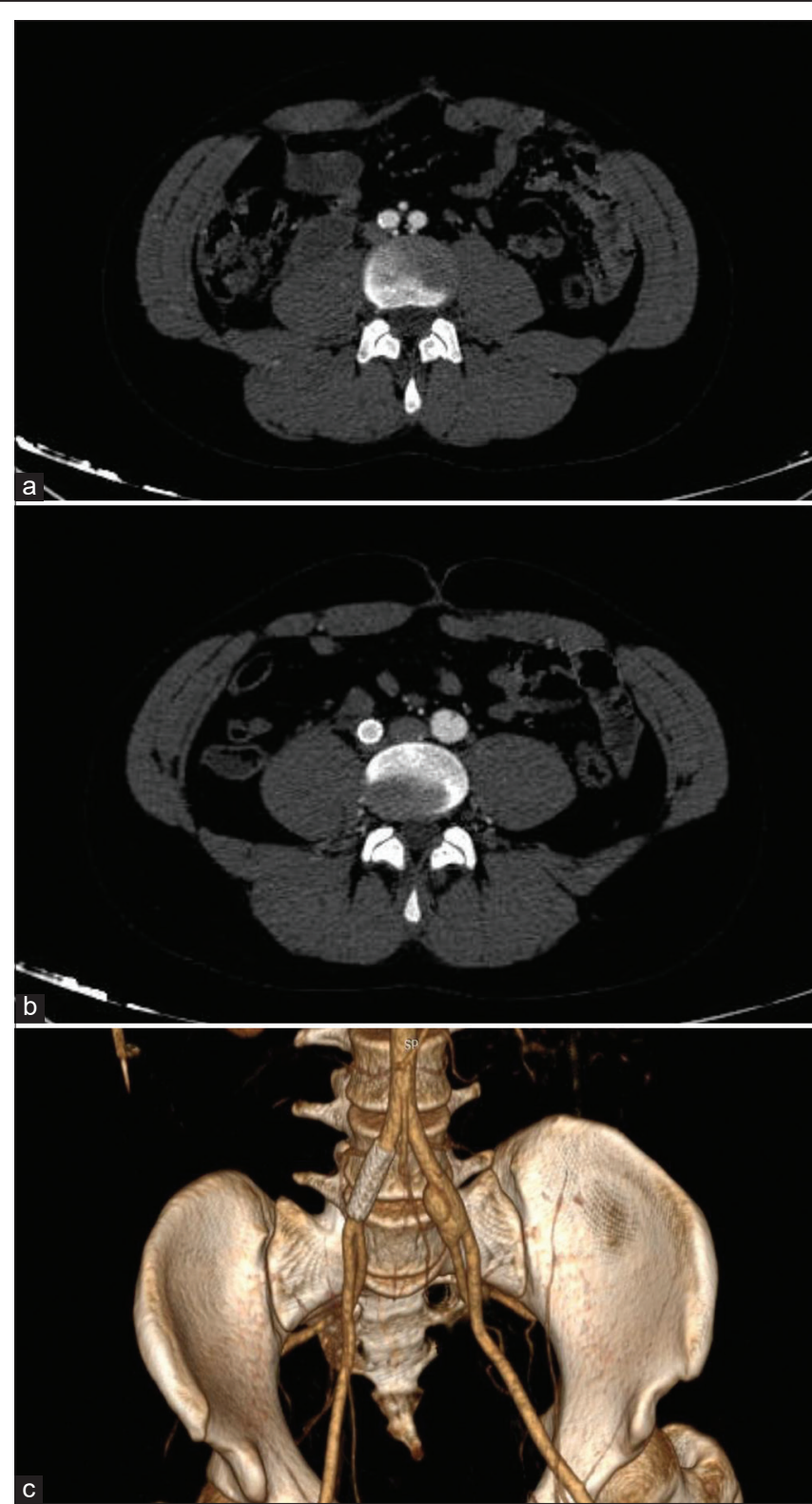

Figure 5: Control CT angiography 1 month later (a) and (b) showing excellent stent patency with almost complete resolution of the retroperitoneal hematoma and (c) VR reconstruction confirms good stent position with persistent pseudoaneurysm of the left CIA

At the follow-up CT angiography, 5 weeks after treatment there was almost complete resorption of the hematoma, with small residual seroma on the right side, complete patency of the stent graft, and persistent pseudoaneurysm of left CIA (Figure 5).

Since the patient had positive family history, was quite young and reported no recent trauma or surgeries, a connective tissue disorder was suspected. He was further sent for hereditary genetic diseases examinations. Subsequently, EDS-IV was diagnosed by identification of abnormal type III procollagen by fibroblast cell culture and gel electrophoresis. The diagnosis was completed by identification of a point mutation in the COL3A1 gene (c. $1662+1 \mathrm{G}>\mathrm{A})$. 


\section{Conclusions}

Vascular repair, whether open or endovascular on EDS-IV patients carries significant risk and should be indicated very carefully. In a 30-year experience with an EDS-IV cohort of 31 patients from the Mayo Clinic, $70 \%$ of vascular operations were performed for urgent indications. Major bleeding complications occurred with $37 \%$ of interventions, and $10 \%$ of patients died as a direct result of hemorrhage [3]. Other series report even higher operative mortalities of $19 \%$ and $41 \%$. [1,5].

In the general population, the presence of an iliac aneurysm portends a high risk of rupture; therefore, elective repair above a threshold of $3 \mathrm{~cm}$ is advocated. Both open and endovascular repair of iliac aneurysms can be accomplished with reasonably low morbidity and mortality [6]. A higher threshold for any vascular surgery in the EDS-IV population has been advocated.

A report of patients treated electively at Johns Hopkins Hospital from 1994 to 2009 reveals a great success, giving a strong reason to consider elective intervention in patients with EDS IV, before rupture happens [2].

The traditional recommendations when dealing with vascular complications of EDS-IV favor conservative approach, with operative treatment being reserved for patients presenting with imminent or frank life-threatening bleeding. However, interventionalists and surgeons are occasionally faced with the dilemma of whether the elective repair of a large asymptomatic aneurysm is indicated or should be reserved only for urgent cases like this one. Decision-making can be difficult because EDS-IV patients have a very heterogeneous spectrum of disease.

There are more than 400 mutations identified in the COL3A1 gene resulting in EDS-IV and the penetrance is nearly $100 \%$, still the phenotypic expression varies in severity $[1,2]$.

Our patient's iliac pseudoaneurysm was successfully treated endovascularly with a covered stent. In the Mayo series, no patient was treated with endovascular techniques other than coil embolization [3]. There have been only few cases with endovascular approach in EDS-IV patients reported, one is an elective case of nonruptured iliac aneurysm treated with EVAR [4], and the other is a case of covered stent treatment for a hepatic artery aneurysm in EDS-IV patient [7].

Despite the technical and clinical success in this single case, taking into consideration that our patient was treated emergently without a diagnosis of the underlying genetic condition, we urge caution before proceeding with endovascular repair in EDS-IV patients. The risk of remote vessel dissection and access-site complications may be significant and difficult to manage.

Several aspects of this case are worth to mention. First, the treatment was necessary to be carried out urgently because of active bleeding. Second, the position and size of the pseudoaneurysm allowed using only one covered stent with specific size which was sufficient for a successful completion of treatment and preserving the IIA. Other endovascular options were also discussed and this one chosen as a most appropriate one regarding the size and shape of the pseudoaneurysm.

Ultimately, patient risk-to benefit assessment compels any decision to proceed with the type of treatment.

Endovascular treatment for EDS-IV patients is feasible and should be considered as an alternative to open surgery in some challenging cases as this one.

\section{References}

1. Pepin M, Schwarze U, Superti-Furga A, Byers PH. Clinical and genetic features of Ehlers-Danlos syndrome type IV, the vascular type. N Engl J Med. 2000;342(10):673-80.https://doi. org/10.1097/00006254-200008000-00004

PMid:10706896

2. Sidawy AN, Perler BA. Rutherford's Vascular Surgery and Endovascular Therapy. $9^{\text {th }}$ ed., Vol. 2. Amsterdam: Elsevier; 2019.

3. Oderich GS, Panneton JM, Bower TC, Lindor NM, Cherry KJ, Noel AA, et al. The spectrum, management and clinical outcome of Ehlers-Danlos type IV: A 30-year experience. J Vasc Surg. 2005;42(1):98-106.https://doi.org/10.1016/j.jvs.2005.03.053 PMid: 16012458

4. Tonnessen BH, Sternbergh WC, Mannava K, Money SR. Endovascular repair of an iliac artery aneurysm in a patient with Ehlers-Danlos syndrome type IV. J Vasc Surg. 2007;45(1):1779. https://doi.org/10.1016/j.jvs.2006.08.071PMid:17210404

5. Cikrit DF, Miles JH, Silver D. Spontaneous arterial perforation: The Ehlers-Danlos specter. J Vasc Surg. 1987;5(2):248-55. https://doi.org/10.1067/mva.1987.avs0050248 PMid:3546738

6. Krupski WC, Selzman CH, Floridia R, Strecker PK, Nehler MR, Whitehill TA. Contemporary management of isolated iliac aneurysms. J Vasc Surg. 1998;28(1):1-11.https://doi. org/10.1016/s0741-5214(98)70194-6

PMid:9685125

7. Casana R, Nano G, Dalainas I, Tealdi DG. Endovascular treatment of hepatic artery aneurysm in a patient with EhlersDanlos syndrome. Case report. Int Angiol. 2004;23(3):291-295. PMid: 15765047

8. Lum YW, Brooke BS, Arnaoutakis GJ, Williams TK, Black JH $3^{\text {rd }}$. Endovascular procedures in patients with Ehlers-Danlos syndrome: Areview of clinical outcomes and iatrogenic complications. Ann Vasc Surg. 2012;26(1):25-33. https://doi.org/10.1016/j.avsg.2011.05.028 PMid:21945330

9. Wyers MC, Schermerhorn ML, Fillinger MF, Powell RJ, Rzucidlo EM, Walsh DB, et al. Internal iliac occlusion without coil embolization during endovascular abdominal aortic aneurysm repair. J Vasc Surg. 2002;36(6):1138-45. https://doi. org/10.1067/mva.2002.129639

PMid: 12469044

10. Schwarze U, Schievink WI, Petty E, Jaff MR, BabovicVuksanovic D, Cherry KJ, et al. Haploinsufficiency for one COL3A1 allele of type III procollagen results in a phenotype similar to the vascular form of Ehlers-Danlos syndrome type IV. Am J Hum Genet. 2001;69(5):989-1001. https://doi.org/10.1086/324123 PMid:11577371 
Author Queries???

AQ5: Kindly cite reference 8-10 in the text part and also cite choronological order 